The Astrophysical JouRNAL, 494:753-758, 1998 February 20

(C) 1998. The American Astronomical Society. All rights reserved. Printed in U.S.A.

\title{
ABSORPTION DIPS IN THE LIGHT CURVES OF GRO J1655-40 AND 4U 1630-47 DURING OUTBURST
}

\author{
Erik KuUlKers, ${ }^{1}$ Rudy Wijnands, ${ }^{2}$ Tomaso Belloni, ${ }^{2}$ Mariano Méndez, ${ }^{2,3}$ \\ MICHIEL VAN DER KLIS, ${ }^{2}$ AND JAN VAN PARADIJS ${ }^{2,4}$ \\ Received 1997 August 11; accepted 1997 September 29
}

\begin{abstract}
Using the RXTE PCA, we discovered deep dips in the X-ray light curves of the black hole candidates GRO J1655-40 and 4U 1630-47 during outburst. Similar kind of dips for GRO J1655-40 were found in $90 \mathrm{~s}$ measurements of the RXTE ASM during the same outburst. The duration of the dips in both sources is of the order of minutes. The occurrences of the dips observed with the RXTE PCA and ASM in GRO J1655-40 are consistent with the optically determined orbital period, and were found between photometric orbital phases 0.72 and 0.86 . This constitutes the first evidence for orbital variations in $\mathrm{X}$-rays for GRO J1655-40. The PCA data indicate that an absorbing medium is responsible for these dips. The X-ray spectra during the dips can be best described by a heavily absorbed component and an unabsorbed component. In the case of GRO J1655-40 we are able constrain the extent of the absorbing medium and the central X-ray source.
\end{abstract}

Subject headings: accretion, accretion disks - binaries: close - black hole physics stars: individual (GRO J1655-40, 4U 1630-47) - X-rays: stars

\section{INTRODUCTION}

$\mathrm{X}$-ray intensity dips caused by an intervening medium have now been found in the light curves of various lowmass and high-mass X-ray binaries (e.g., Parmar \& White 1988; Marshall et al. 1993; Saraswat et al. 1996, and references therein). During the majority of these dips the X-ray spectra harden, which is indicative of photoelectric absorption of radiation from the central X-ray source. However, a simple neutral and uniform medium which absorbs the emission does not fit the X-ray spectra. Instead, the spectra reveal an excess flux at low energies (typically $\lesssim 4 \mathrm{keV}$ ) compared to that expected from the amount of absorption estimated from data above $\sim 4 \mathrm{keV}$.

In this paper we report on such dips seen in the X-ray light curves of the black hole (candidate) soft X-ray transients GRO J1655 - 40 and 4U 1630 - 47 during their 1996/ 1997 and 1996 outbursts, respectively, as obtained with the Proportional Counter Array (PCA) and the All Sky Monitor (ASM) on board the Rossi X-Ray Timing Explorer (RXTE).

GRO J1655-40 was discovered during an outburst in 1994 and has since shown irregular outburst activity (e.g., Zhang et al. 1997). Dynamical measurements suggest that the compact star in GRO J1655-40 is a black hole, with a mass of $\sim 7 M_{\odot}$ (Orosz \& Bailyn 1997; van der Hooft et al. 1998). Recently, Ueda et al. (1998) found evidence for a $\sim 5.7 \mathrm{hr}$ dip down to $\sim 25 \%$ of the out-of-dip intensity in the ASCA light curve of GRO J1655-40, with no clear evidence of spectral hardening. The X-ray dips reported

\footnotetext{
${ }^{1}$ Astrophysics, University of Oxford, Nuclear and Astrophysics Laboratory, Keble Road, Oxford OX1 3RH, United Kingdom; erik@astro.ox.ac.uk.

2 Astronomical Institute "Anton Pannekoek," University of Amsterdam and Center for High-Energy Astrophysics, Kruislaan 403, NL-1098 SJ Amsterdam, Netherlands; rudy@astro.uva.nl, tmb@astro.uva.nl, mariano@astro.uva.nl,michiel@astro.uva.nl,jvp@astro.uva.nl.

${ }^{3}$ Facultad de Ciencias Astronómicas y Geofisicas, Universidad Nacional de La Plata, Paseo del Bosque S/N, 1900 La Plata, Argentina.

${ }^{4}$ Physics Department, University of Alabama at Huntsville, Huntsville, AL 35899.
}

here are considerably deeper (down to $\sim 8 \%$ of the out-ofdip intensity) and have much shorter duration (of the order of minutes). A preliminary announcement of the dips in GRO J1655-40 observed by RXTE has already been given by Kuulkers et al. (1997a).

$4 \mathrm{U} 1630-47$ has shown outbursts every $\sim 600$ days since at least 1969 (e.g., Kuulkers et al. 1997b). The nature of the compact star in $4 \mathrm{U} 1630-47$ is unknown. Its X-ray spectral (e.g., Barret, McClintock, \& Grindlay 1996) and X-ray timing (Kuulkers, van der Klis, \& Parmar 1997c) properties during outburst suggest it is a black hole. Recently, Kuulkers et al. (1997b, 1997c) pointed out similarities in X-ray behavior between $4 \mathrm{U} 1630-47$ and GRO J1655-40, and postulated that they are systems of a similar kind.

\section{OBSERVATIONS AND ANALYSIS}

The RXTE PCA (Bradt, Rothschild, \& Swank 1993) performed public Target of Opportunity observations of $4 \mathrm{U}$ $1630-47$ on UTC 1996 May 3, 20:49-22:41, and of GRO J1655-40 on UTC 1997 February 26, 19:34-23:30. The data were collected with a time resolution of $16 \mathrm{~s}(129$ photon energy channels, covering $2.0-60 \mathrm{keV})$ and $125 \mu \mathrm{s}(3$ energy channels, covering 2.0-5.0-8.7-13.0 keV). The $16 \mathrm{~s}$ data were used in the X-ray spectral analysis. From the high time resolution data we constructed light curves by rebinning the "raw" (i.e., not corrected for dead time) background-subtracted count rates to a time resolution of $0.25 \mathrm{~s}$, and hardness values using count-rate ratios binned to a time resolution of $0.25 \mathrm{~s}$ (GRO J1655-40) or $0.5 \mathrm{~s} \mathrm{(4 \textrm {U }}$ 1630-47). All X-ray spectral fits were performed in the range $2-30 \mathrm{keV}$ (out of dip) or $2-20 \mathrm{keV}$ (dip), and a $2 \%$ uncertainty was included in the data to account for uncertainties in the PCA response matrix (see, e.g., Belloni et al. 1997). The X-ray spectra were corrected for background and dead time. Errors quoted for the spectral parameters were determined using $\Delta \chi^{2}=2.706(2 \sigma)$.

The ASM (Levine et al. 1996) on board RXTE scans the sky in series of $90 \mathrm{~s}$ dwell times in three energy bands, 1.5-3, $3-5$, and $5-12 \mathrm{keV}$. Because of satellite motion and a $\sim 40 \%$ duty cycle, any given source is scanned 5-10 times per day. 
For our analysis we used the results provided by the $R X T E$ ASM team, covering the period from 1996 February 21 to 1997 June 19.

\section{RESULTS}

\subsection{Intensity and Hardness Variations}

The $2.0-13.0 \mathrm{keV}$ count rates of GRO J1655-40 and $4 \mathrm{U}$ $1630-47$ were generally $\sim 11,500$ and $\sim 3000$ counts $\mathrm{s}^{-1}$, respectively. However, both sources showed sharp, deep drops in their count rates down to $\sim 1000$ counts $\mathrm{s}^{-1}$, lasting $\sim 50-60 \mathrm{~s}$ for GRO J1655-40 (two events), and $\sim 140$ s for $4 \mathrm{U} 1630-47$ (one event), preceded and followed by short dips (Fig. 1, upper panel). We will refer to the out-of-dip count rate as the "persistent emission" (PE).

The fall timescales of the main dips of GRO J1655-40 are 2-4 s, while the rise timescales are 3-5 s. The hardness curve (Fig. 1, lower left panel) and the corresponding hardness versus intensity diagram (HID; Fig. 2, upper left panel) of GRO J1655-40 show that as the intensity drops the $\mathrm{X}$-ray spectrum becomes much harder, until a certain threshold count rate of $\sim 2000$ counts $\mathrm{s}^{-1}$ is reached. During the last part of the fall, the spectrum softens again to almost the same value as the PE level. The reverse behavior is seen during the rise of the dips. In the HID the source always follows the same loop, also during the small pre- and afterdips and during the short spike in the second dip.

The fall and rise timescales of the main dip of $4 \mathrm{U}$ $1630-47$ are $\sim 3 \mathrm{~s}$. During the main dip several small flares occurred. The light curve and hardness behavior (Fig. 1, upper right panel; Fig. 2, lower panel) of 4U 1630-47 resembles that of GRO J1655-40. Below a certain threshold count rate the hardness is positively correlated with intensity. However, the change in hardness between the persistent and threshold count rate is much smaller than that observed in GRO J1655-40. Below the threshold count rate the hardness drops below the persistent value.

In Figure 3 we plot the RXTE ASM light curve of the individual $90 \mathrm{~s}$ dwell times for GRO J1655-40. Twelve clear drops in the $2-12 \mathrm{keV}$ intensity can be seen down by $\sim 25 \%-95 \%$. During these dips the spectral hardness increases. The occurrences of the ASM and PCA dips (Table 1) are best fitted with a period of $2.6213 \pm 0.0005$ days $(1 \sigma)$. All these dips occurred between photometric orbital phases 0.72 and 0.86 (Table 1 ).

\subsection{Energy Spectra}

We constructed a series of $\sim 200 \mathrm{~s} X$-ray spectra just outside the dips, and individual $16 \mathrm{~s}$ spectra at the bottom of the dips. Because of the $16 \mathrm{~s}$ time resolution we could not

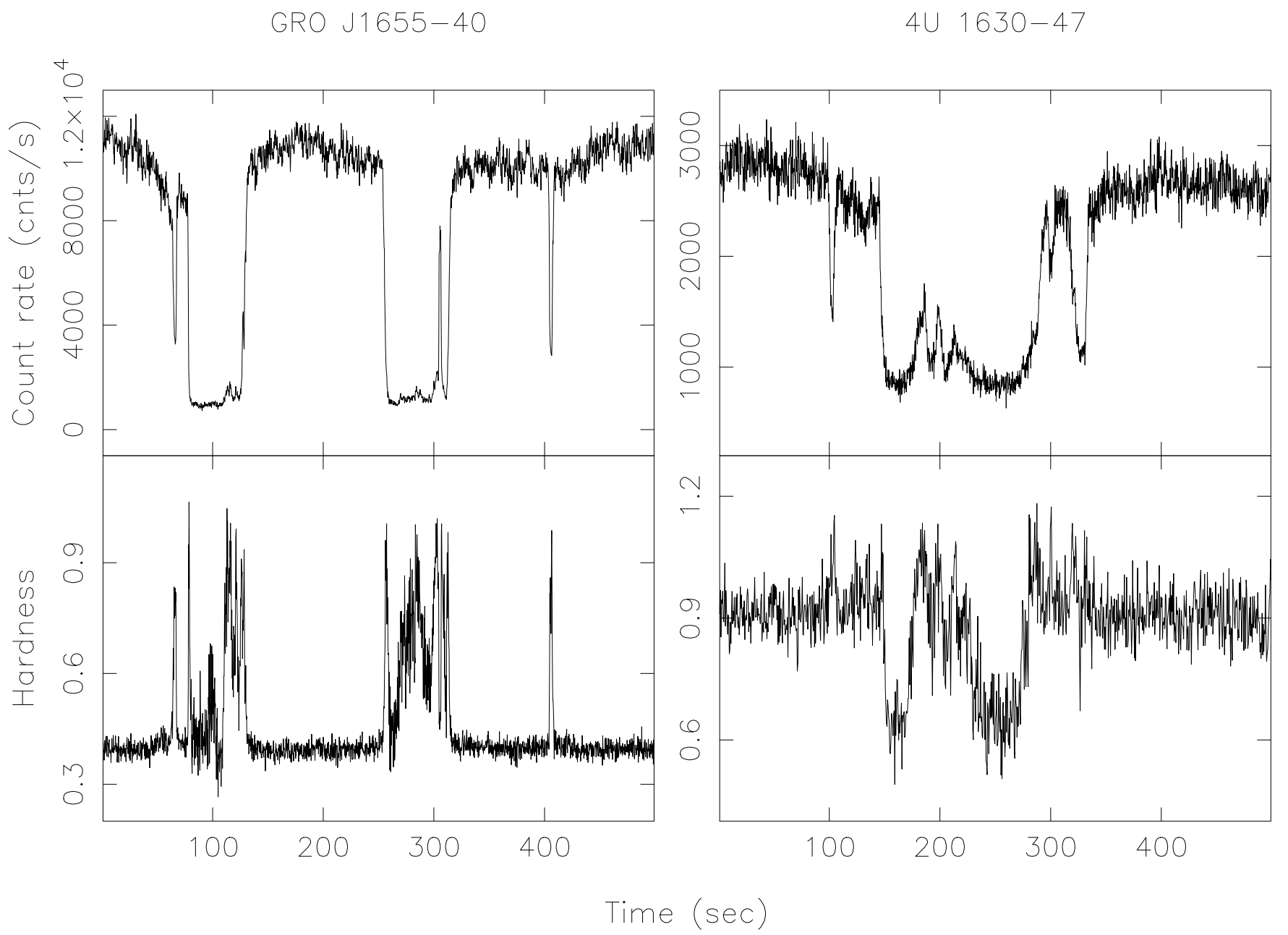

FIG. 1.-Light curves (upper panels) and hardness curves (lower panels) of GRO J1655-40 (left panels) and 4U $1630-47$ (right panels). Hardness is defined as the ratio of the count rates in the $5.0-13.0 \mathrm{keV}$ and $2.0-5.0 \mathrm{keV}$ bands. The time resolution in all panels is $0.25 \mathrm{~s}$, except for the lower right panel, where it is $0.5 \mathrm{~s} . T=0 \mathrm{~s}$ corresponds to UTC 1997 February 26, 21:22:21, for GRO J1655 - 40 and UTC 1996 May 3, 20:48:46, for 4U 1630-47. 

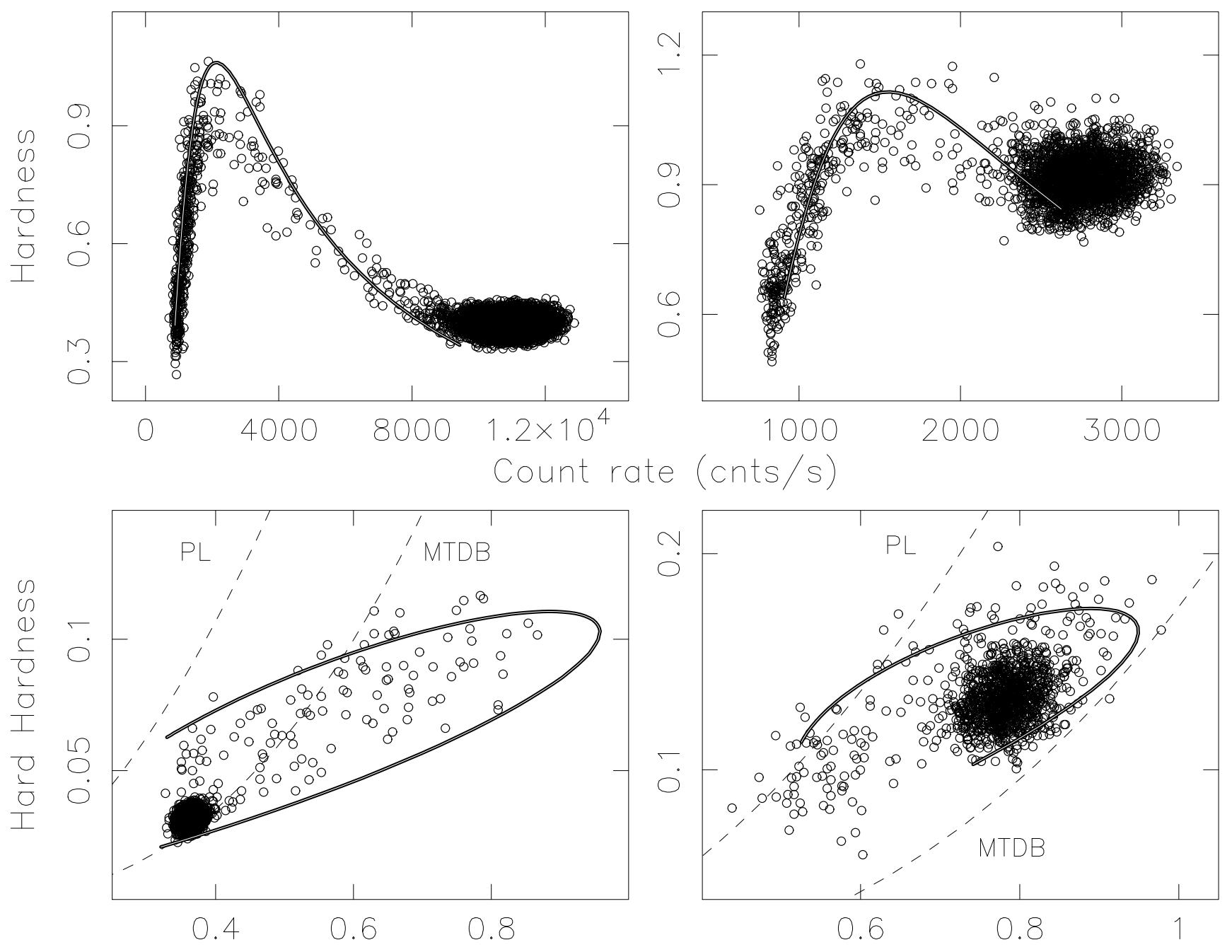

\section{Soft Hardness}

FIG. 2.-Upper panel: Hardness-intensity diagrams for GRO J1655-40 ( $0.25 \mathrm{~s}$ time resolution; left) and $4 \mathrm{U} 1630-47$ ( $0.5 \mathrm{~s}$ time resolution; right), where hardness is defined as in Fig. 1. The line through the data points corresponds to a simple model of the X-ray spectral behavior as described in the text. Lower panel: ratio of the count rates in the 8.7-13.0 keV and 2.0-5.0 keV bands ("hard hardness") versus the ratio of the count rates in the 5.0-8.7 keV and 2.0-5.0 $\mathrm{keV}$ bands ("soft hardness") for GRO J1655-40 (left) and 4U 1630-47 (right). The data points (1 s averages) are shown as open circles. The upper left dashed line is for the power-law component, and the lower right dashed line is for the disk blackbody component. The line through the data points corresponds to a simple model of the X-ray spectral behavior as described in the text.

TABLE 1

GRO J1655 - 40 DIP OCCURRENCE TIMES

\begin{tabular}{cccccc}
\hline \hline $\begin{array}{c}\text { Time } \\
(\mathrm{JD}-2,450,000)\end{array}$ & $\phi_{\text {orb }}{ }^{\mathrm{a}}$ & $(\mathrm{JD}-2,450,000)$ & $\phi_{\text {orb }}{ }^{\mathrm{a}}$ & $(\mathrm{JD}-2,450,000)$ & $\phi_{\text {orb }}{ }^{\mathrm{a}}$ \\
\hline 205.0796 & 0.86 & 225.2172 & 0.73 & 487.4261 & 0.75 \\
220.0827 & 0.78 & 225.2183 & 0.73 & 487.4909 & 0.78 \\
220.0850 & 0.78 & 228.1535 & 0.85 & $505.9143^{\mathrm{b}}$ & 0.81 \\
222.6122 & 0.74 & 228.1546 & 0.85 & $505.9163^{\mathrm{b}}$ & 0.81 \\
222.8194 & 0.82 & 254.3466 & 0.85 & 555.7916 & 0.83 \\
222.8205 & 0.82 & 280.2353 & 0.72 & 555.7927 & 0.83 \\
222.8681 & 0.84 & 296.2989 & 0.85 & 565.9994 & 0.73 \\
222.8692 & 0.84 & 314.4044 & 0.75 & $\ldots$ & $\cdots$ \\
\hline
\end{tabular}

${ }^{\text {a }}$ Photometric orbital phase (Orosz \& Bailyn 1997).

${ }^{\mathrm{b}}$ RXTE PCA; see $\S 3.1$. 
TABLE 2

Persistent Emission: X-RAy SpeCtral Fit Results ${ }^{a}$

\begin{tabular}{|c|c|c|c|c|c|c|c|}
\hline Source & $\begin{array}{c}\bar{I} \\
\left.\text { (counts s}^{-1}\right)\end{array}$ & 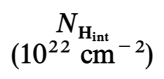 & $\begin{array}{c}T_{\text {in }} \\
(\mathrm{keV})\end{array}$ & $\begin{array}{r}R_{\mathrm{in}} \\
(\mathrm{km})\end{array}$ & $\Gamma$ & $\underset{\left(\mathrm{cm}^{-2} \mathrm{~s}^{-1} \mathrm{keV}^{-1}\right)}{ }$ & $\chi_{v}^{2} / \mathrm{dof}$ \\
\hline GRO J1655-40..... & 10807 & $2.17 \pm 0.11$ & $1.110 \pm 0.002$ & $24.7 \pm 1.0^{b}$ & $2.48 \pm 0.02$ & $0.29 \pm 0.01$ & $1.6 / 54$ \\
\hline $4 \mathrm{U} 1630-47 \ldots \ldots \ldots$ & 2759 & $12.3 \pm 0.3$ & $1.277 \pm 0.005$ & $17.9 \pm 0.3^{\mathrm{c}}$ & $3.65 \pm 0.12$ & $26 \pm 8$ & $2.0 / 54$ \\
\hline
\end{tabular}

${ }^{\text {a }}$ Model: $\exp \left[-N_{\mathrm{H}_{\mathrm{int}}} \sigma(E)\right]\left[A_{\mathrm{pl}} E^{-\Gamma}+\mathrm{DBB}\left(T_{\mathrm{in}}, R_{\mathrm{in}}\right)\right]$, where $N_{\mathrm{H}_{\mathrm{int}}}$ is the interstellar absorption column density, $E$ is the photon energy, and DBB is the disk-blackbody model. Errors were determined using $\Delta \chi^{2^{\mathrm{nt}}}=2.706(2 \sigma)$.

${ }^{b}$ Assuming a distance of $3.2 \mathrm{kpc}$ (Hjellming \& Rupen 1995) and an inclination of 70 (Orosz \& Bailyn 1997; van der Hooft et al. 1997).

${ }^{\mathrm{c}}$ In units of $(\cos i)^{-1 / 2} d_{10}$, where $i$ is the inclination of the accretion disk and $d_{10}$ is the distance in units of $10 \mathrm{kpc}$.

accumulate spectra during the rise and fall of the dips. The average count rates during the dip spectra are all below the threshold count rate.

Fits to the PE with simple single-component models did not give acceptable results. We therefore used the model usually employed for black hole candidates, i.e., a diskblackbody (DBB) plus a power law (Mitsuda et al. 1984), which gave values of $\chi_{v}^{2} \lesssim 2$. Using the mean spectral fits (Table 2), we find that the persistent 2-30 keV X-ray flux was $\sim 2.5 \times 10^{-8}$ and $\sim 5.8 \times 10^{-9} \mathrm{ergs} \mathrm{cm}^{-2} \mathrm{~s}^{-1}$, for GRO J1655-40 and 4U 1630-47, respectively.

The spectral hardening between the PE count rate and the threshold value suggests that absorption is involved in the process giving rise to the dips. To verify this, we also made color-color diagrams (CDs; see Fig. 2, lower panels). The data points for the two sources during the dips move from the crowded regions (PE) to the upper right, and then bend back to the lower left part of the CD (in the case of $4 \mathrm{U}$ $1630-47$ the data points bend back almost immediately). The bend-over point corresponds to the threshold count rate. We also plotted the calculated hardness values for the DBB and power-law component separately for a range of values of the inner disk temperature, $T_{\text {in }}$, and of the powerlaw index, $\Gamma$, with interstellar absorption column density, $N_{\mathrm{H}_{\text {int }}}$, fixed to the persistent value (Fig. 2, dashed lines). With our choice of hardness ratios any linear combination of the

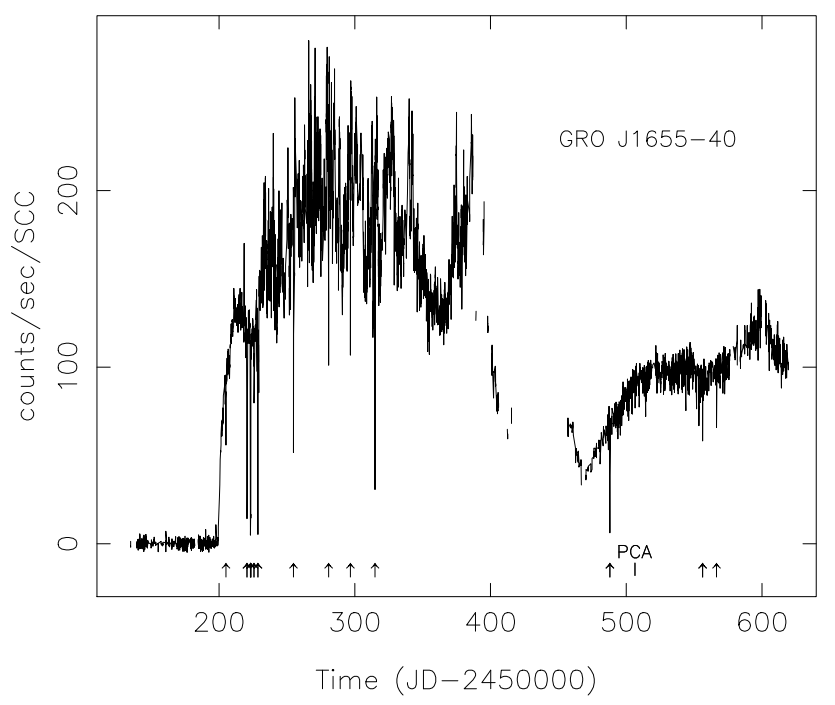

FIG. 3.- RXTE ASM light curve of GRO J1655-40 of data from individual dwell times of $\sim 90$ s from 1996 February 21 (JD 2,450,135) to 1997 June 19 (JD 2,450,618). Data points separated by less than 2 days have been connected to guide the eye. Clearly, deep sharp dips can be seen. The arrows at the bottom denote the dips used in the text. Indicated also is the time of the dips observed with the PCA. two components has to lie between these two lines. Only in the CD of GRO J1655-40 does part of the loop lie below the DBB line; this requires an increase in the absorption column.

Homogeneous absorption of the persistent DBB and power-law components by cold material did not fit the dip spectra well, especially at low energies. The observed flux below $\sim 6 \mathrm{keV}$ is much in excess of that expected in this

TABLE 3

Dips: X-RAy Spectral Fit Results

\begin{tabular}{|c|c|c|}
\hline \multirow[b]{2}{*}{ PARAMETER } & \multicolumn{2}{|c|}{ DIP $^{a}$} \\
\hline & GRO J1655-40 & $4 U 1630-47$ \\
\hline \multicolumn{3}{|c|}{ Power Law + Absorbed DBB ${ }^{b}$} \\
\hline$N_{\mathrm{H}_{\text {int }}}\left(10^{22} \mathrm{~cm}^{-2}\right) \ldots \ldots \ldots \ldots$ & $2.17^{\mathrm{c}}$ & $12.3^{\mathrm{c}}$ \\
\hline$\gamma \ldots \ldots \ldots \ldots \ldots \ldots \ldots \ldots \ldots \ldots \ldots \ldots \ldots$ & $4.6_{-0.2}^{+0.8}$ & $4.5 \pm 0.1$ \\
\hline$A_{\mathrm{pl}}\left(\mathrm{cm}^{-2} \mathrm{~s}^{-1} \mathrm{keV}^{-1}\right) \ldots \ldots$ & $34_{-6}^{+35}$ & $73_{-18}^{+10}$ \\
\hline$N_{\mathrm{H}}\left(10^{22} \mathrm{~cm}^{-2}\right) \ldots \ldots \ldots \ldots \ldots$ & $27_{-18}^{+26}$ & $34 \pm 27$ \\
\hline$T_{\text {in }}(\mathrm{keV}) \ldots \ldots \ldots \ldots \ldots \ldots$ & $1.1 \pm 0.2$ & $0.9 \pm 0.1$ \\
\hline$R_{\mathrm{in}}^{\mathrm{d}}(\mathrm{km}) \ldots \ldots \ldots \ldots \ldots \ldots$ & $7_{-3}^{+18}$ & $17.9^{\mathrm{c}}$ \\
\hline$\chi_{v}^{2} /$ dof $\ldots \ldots \ldots \ldots \ldots \ldots \ldots \ldots$ & $2.0 / 41$ & $1.2 / 42$ \\
\hline \multicolumn{3}{|c|}{ Blackbody (BB) + Absorbed DBB ${ }^{\mathrm{e}}$} \\
\hline$N_{\mathrm{H}_{\text {int }}}\left(10^{22} \mathrm{~cm}^{-2}\right)$. & $2.17^{\mathrm{c}}$ & $12.3^{\mathrm{c}}$ \\
\hline$T_{\mathrm{bb}}(\mathrm{keV}) \ldots \ldots \ldots \ldots \ldots \ldots$ & $0.60 \pm 0.04$ & $0.60 \pm 0.03$ \\
\hline$R_{\mathrm{bb}}{ }^{\mathrm{f}}(\mathrm{km}) \ldots \ldots \ldots \ldots \ldots \ldots$ & $34 \pm 5$ & $49 \pm 6$ \\
\hline$N_{\mathrm{H}}\left(10^{22} \mathrm{~cm}^{-2}\right) \ldots \ldots \ldots \ldots \ldots$ & $79_{-27}^{+49}$ & $76_{-30}^{+37}$ \\
\hline$T_{\text {in }}(\mathrm{keV}) \ldots \ldots \ldots \ldots \ldots \ldots \ldots$ & $1.1 \pm 0.1$ & $1.4 \pm 0.2$ \\
\hline$R_{\text {in }}(\mathrm{km}) \ldots \ldots \ldots \ldots \ldots \ldots$ & $15_{-5}^{+31}$ & $12_{-4}^{+14}$ \\
\hline$\chi_{v}^{2} /$ dof $\ldots \ldots \ldots \ldots \ldots \ldots \ldots \ldots$ & $1.4 / 41$ & $1.6 / 41$ \\
\hline \multicolumn{3}{|c|}{ Partial Covering Absorption of $\mathrm{DBB}^{\mathrm{g}}$} \\
\hline$N_{\mathrm{H}_{\text {int }}}\left(10^{22} \mathrm{~cm}^{-2}\right)$. & $2.17^{\mathrm{c}}$ & $5.7 \pm 1.0$ \\
\hline$F \ldots \ldots \ldots \ldots \ldots \ldots$ & $0.985 \pm 0.007$ & $0.97 \pm 0.01$ \\
\hline$N_{\mathrm{H}}\left(10^{22} \mathrm{~cm}^{-2}\right) \ldots \ldots \ldots \ldots$ & $191 \pm 17$ & $299_{-40}^{+77}$ \\
\hline$T_{\text {in }}(\mathrm{keV}) \ldots \ldots \ldots \ldots \ldots \ldots$ & $0.89 \pm 0.03$ & $1.19 \pm 0.07$ \\
\hline$R_{\text {in }}(\mathrm{km}) \ldots \ldots \ldots \ldots \ldots \ldots$ & $100 \pm 29$ & $67_{-15}^{+35}$ \\
\hline$\chi_{v}^{2} /$ dof $\ldots \ldots \ldots \ldots \ldots \ldots \ldots$ & $1.7 / 42$ & $1.2 / 41$ \\
\hline
\end{tabular}

${ }^{\text {a }}$ GRO J1655-40 dip: $\bar{I}=878$ counts $\mathrm{s}^{-1} ; 4 \mathrm{U} 1630-47$ dip: $\bar{I}=860$ counts $\mathrm{s}^{-1}$.

b Model: $\exp \left[-N_{\mathrm{H}_{\text {int }}} \sigma(E)\right]\left\{A_{\mathrm{pl}} E^{-\gamma}+\exp \left[-N_{\mathrm{H}} \sigma(E)\right]\left[\mathrm{DBB}\left(T_{\mathrm{in}}\right.\right.\right.$,

$\left.\left.\left.R_{\mathrm{in}}\right)\right]\right\}$, where $\sigma(E)$ is the photoelectric absorption cross section.

${ }^{c}$ Parameter fixed to the persistent emission value.

${ }^{\mathrm{d}}$ In the case of $4 \mathrm{U} 1630-47$ in units of $(\cos i)^{-1 / 2} d_{10}$; see also Table 2.

e Model: $\exp \quad\left[-N_{\mathrm{H}_{\text {int }}} \sigma(E)\right]\left\{\mathrm{BB}\left(T_{\mathrm{bb}}, \quad R_{\mathrm{bb}}\right)+\exp \quad\left[-N_{\mathrm{H}} \sigma(E)\right]\right.$ $\left.\left[\mathrm{DBB}\left(T_{\text {in }}, R_{\text {in }}\right)\right]\right\}$.

${ }^{f}$ Apparent blackbody radius at $3.2 \mathrm{kpc}$ (GRO J1655-40) or 10 $\operatorname{kpc}(4 \mathrm{U} 1630-47)$.

${ }^{\mathrm{g}}$ Model: $\exp \left[-N_{\mathrm{H}_{\mathrm{int}}} \sigma(E)\right]\left[F e^{-N_{\mathrm{H}} \sigma(E)}+(1-F)\right]\left[\mathrm{DBB}\left(T_{\mathrm{in}}, R_{\mathrm{in}}\right)\right]$, where $F$ is the covering fraction. 
model. We performed simple fits to the spectra by modeling this low-energy excess either as a separate component (power-law or blackbody) or by partial absorption of the persistent components (e.g., Marshall et al. 1993). We find that the persistent power-law component is absent during the dips. We focus on the results of fits to the spectra at the bottom of the dips (Table 3) to investigate the low-energy excess, since there the absorption is expected to be largest. We fixed $N_{\mathrm{H}_{\text {int }}}$ to the values derived from the PE fits, except when using the partial covering absorption model for $4 \mathrm{U}$ $1630-47$ because this led to an unstable fit. When using a power law to model the low-energy excess plus an absorbed DBB for the dip spectrum of $4 \mathrm{U} 1630-47$, the inner disk radius, $R_{\mathrm{in}}$, could not be constrained and was, therefore, fixed to its $P E$ value.

The fits show that the shape and strength of the lowenergy excess are very similar for the two sources, and its contribution is only $6 \%-7 \%$ of the PE flux. When the lowenergy excess is modeled as a separate component, the DBB parameters are consistent with the PE values. When a partial covering model is used to describe the spectra, we get somewhat lower values for $T_{\text {in }}$ with respect to their PE values. Depending on the model, we find that the absorption of the DBB component increased up to $\sim(25-$ 200) $\times 10^{22}$ and $\sim(35-300) \times 10^{22} \mathrm{~cm}^{-2}$ at the lowest mean dip intensities in GRO J1655-40 and 4U 1630-47, respectively.

To see whether we can qualitatively reproduce the observed shapes of the HID and CD, we calculated several sequences of X-ray spectra and determined intensity and hardness values. The out-of-dip spectrum was modeled by the persistent DBB component (only subject to interstellar absorption) as given in Table 2, plus the low-energy excess contribution modeled by a blackbody, as given in Table 3 . In the dip we linearly increased the absorption of the persistent DBB component from zero up to 150 and $300 \times 10^{22} \mathrm{~cm}^{-2}$, for GRO J1655-40 and 4U 1630-47, respectively, fixing the rest of the parameters to those given by the out-of-dip spectrum. The results are plotted in Figure 2, and show that a gradual increase in absorption of the DBB component can reproduce the observed dip behavior.

\section{DISCUSSION}

\subsection{RXTE PCA and ASM Dips}

We have discovered short-term (of the order of minutes) X-ray dipping behavior of GRO J1655-40 and 4U $1630-47$, down to $\sim 8 \%$ and $\sim 30 \%$ of the out-of-dip intensity, respectively. We found similar intensity drops in GRO J1655-40 during 90 s measurements with the $R X T E$ ASM. The duration of the dips is of the same order as that of those found in Cyg X-1 and Her X-1 (e.g., Kitamoto et al. 1984; Leahy 1997), but is shorter than those typically seen in LMXB dip sources (e.g., Parmar \& White 1988).

The best-fit period of the occurrence of the dips observed in GRO J1655-40 is consistent with the optical period of the system (Orosz \& Bailyn 1997; van der Hooft et al. 1998). This therefore constitutes the first evidence of the orbital period in GRO J1655 - 40 in X-rays. All these dips occurred between photometric orbital phases 0.72 and 0.86 . We note that the X-ray dip in GRO J1655-40 seen with $A S C A$ (Ueda et al. 1998) occurred near photometric orbital phase 0.6. The phasing of the occurrence of the X-ray dips is very similar to that observed in the low-mass X-ray binary dip sources (e.g., Parmar \& White 1988), which suggests a similar origin for the cause of the dips. The inclinations of such sources which show only dips and no eclipses are in the range $60^{\circ}-75^{\circ}$ (e.g., Frank, King, \& Lasota 1987). The inclination inferred for GRO J1655-40 (Orosz \& Bailyn 1997; van der Hooft et al. 1998) is in agreement with this. Since the dip behavior of $4 \mathrm{U} 1630-47$ is so similar to that seen in GRO J1655-40, we propose that $4 \mathrm{U} 1630-47$ is also seen at a relatively high inclination, i.e., $60^{\circ}-75^{\circ}$.

\subsection{An Absorbing Medium}

The observed dips are caused by an intervening medium absorbing the out-of-dip (persistent) emission (PE). A simple neutral and uniform medium that heavily absorbs the PE, however, does not describe the spectra during the dips very well. The dip spectra can be best described by a heavily absorbed persistent component, plus a low-energy component that typically appears below $\sim 6 \mathrm{keV}$. Such an extra low-energy component has also been reported by Ueda et al. (1998) in GRO J1655-40 during the dip they observed with $A S C A$, and has been seen during dips and eclipses in other low-mass and high-mass X-ray binaries (§ 1). We modeled this so-called low-energy excess as a power law or a blackbody (subject only to interstellar absorption), or alternatively by partial covering absorption of the persistent components. We find that the maximum absorption of the persistent components at the bottom of the dips $\left[\sim(25-300) \times 10^{22} \mathrm{~cm}^{-2}\right]$ is comparable to that found during the long dip in GRO J1655-40 observed with $A S C A$ (Ueda et al. 1998) and the bottom of deep dips and/or eclipses of other binaries.

Several models have been proposed to explain the lowenergy excess during dips and/or eclipses (e.g., Marshall et al. 1993). These include either a separate component that is not affected by the variable heavy absorption, or partial covering by a clumpy medium. The former has been proposed to be due to a scattering halo (e.g., Kitamoto, Miyamoto, \& Yamamoto 1989), or ionized absorber models, in which the absorbing medium is sufficiently ionized to reduce the soft X-ray absorption (e.g., Parmar \& White 1988; Marshall et al. 1993). We note that Ueda et al. (1998) reported iron absorption lines during $A S C A$ out-of-dip spectra of GRO J1655 - 40, which suggests the presence of highly ionized plasma. Alternatively, the low-energy excess may be the result of accumulating spectra over timescales longer than the intrinsic timescale for variability during the dips (e.g., Parmar et al. 1986).

Our spectral fits indicate that during the dips the persistent power-law component is absent. This suggests not only that the region where the bulk of the $\mathrm{X}$-rays originate is subject to absorption but also that the power-law-emitting region is blocked from our view. Interaction of the inflowing gas stream from the secondary with the outer edge of the disk may cause a thickening of the outer edge and/or material above the disk in the expected $0.6-0.0$ phase range (e.g., Parmar \& White 1988) and may well cause this absorption and shadowing.

Recently, Greiner, Morgan, \& Remillard (1996) showed that the Galactic superluminal source GRS $1915+105$ had "dipping" behavior during its outbursts. The dipping activity in GRS $1915+105$ is much more complex than that seen in our observations of GRO J1655-40 and 4U 1630-47. This dipping behavior has been proposed to be due to 
thermal-viscous instabilities in the inner disk (Belloni et al. 1997), and is therefore not related to the dipping behavior we see in GRO J1655 - 40 and 4U 1630-47.

$$
\text { 4.3. GRO J1655-40 }
$$

For GRO J1655-40 the system parameters have been well determined (Orosz \& Bailyn 1997; van der Hooft et al. 1998). In this system the timescales of the dips impose constraints on the sizes of the different emitting and absorbing media. The fall and rise times $\left(t_{r, f} \sim 3.5 \mathrm{~s}\right)$ constrain the size of the region which is "obscured"; in fact, this gives an upper limit on the size, because the region over which the column density increases significantly also has a finite width (Leahy, Yoshida, \& Matsuoka 1994). The duration of the dips $\left(t_{\mathrm{dip}} \sim 55 \mathrm{~s}\right)$ constrains the size of the absorbing medium itself.

Since $t_{r, f} \ll t_{\text {dip }}$, we may assume that the absorbed X-ray source is much smaller than the absorbing medium. A medium which crosses a pointlike central X-ray source may produce irregular X-ray dips, whereas crossing an extended region such as an accretion disk corona may produce smoother and longer energy-independent modulations (e.g., Parmar \& White 1988). If the medium corotates in the binary frame and is located at a radius which is smaller than the outer disk radius $\left(r_{d} \sim 0.85 R_{L}\right.$ [Orosz \& Bailyn 1997], where $R_{L}$ is the effective Roche lobe radius of the black hole) the upper limit on the size of the X-ray-emitting region is $\sim 460 \mathrm{~km}$. If the medium corotates with matter in the accretion disk (i.e., with a Kepler velocity), the upper limit becomes $\sim 2000 \mathrm{~km}$. Similar reasoning gives an approximate upper limit on the size of the absorbing medium of $\sim 7200 \mathrm{~km}$ or $\sim 32,000 \mathrm{~km}$, in the case of corotation in the binary frame or rotation within the accretion disk.

If partial ionization of material in the disk causes the low-energy excess during the dips, one can roughly estimate the location of the absorbing material (e.g., Parmar et al. 1986). For the material causing the intensity dips to be significantly ionized, the ionization parameter $\xi=L / n R^{2}$ (where $L$ is the central source luminosity, $n$ is the gas density of the cloud, and $R$ is the distance from the central source to the clouds) must be larger than $\sim 100$ ergs $\mathrm{cm} \mathrm{s}^{-1}$ (see Hatchett, Buff, \& McCray 1976). Using a typical column density of $\sim 100 \times 10^{22} \mathrm{~cm}^{-2}$ at the bottom of the dips, an out-of-dip luminosity of $L \simeq 3 \times 10^{37} \mathrm{ergs} \mathrm{s}^{-1}$ (at a distance of $3.2 \mathrm{kpc}$; Hjellming \& Rupen 1995), and following Parmar et al. (1986), we derive the result that the material closer than $\sim 3 \times 10^{5} \mathrm{~km}$ from the X-ray source will be significantly ionized. Assuming that the absorbing medium is more or less spherical and has a fixed position in the binary system, one gets (e.g., Remillard \& Canizares 1984) $\xi=2 \pi L t_{\text {dip }}\left(N_{\mathrm{H}} P_{\text {orb }} R\right)^{-1} \gtrsim 100$, and therefore $R \lesssim 5000$ $\mathrm{km}$. So, if ionization plays a role, the absorbing medium should be located in the inner part of the disk. Frank et al. (1987) suggested that the low-energy excess is caused by scattering of X-rays in hot clouds that are the result of material from the accretion stream which skimmed over the disk and formed a ring near the central X-ray source. Ionization may generate a two-phase medium which produces the dips. The expected circularization radius of the ring of material in this model is rather large for GRO J1655-40 $\left(\sim 1 \times 10^{6} \mathrm{~km}\right)$, so that material in this ring cannot be easily ionized.

We thank Phil Charles and various participants of the Microquasar Workshop (1997 May 1-3, GSFC, Greenbelt, MD) for stimulating discussions. Keith Jahoda is acknowledged for providing an up-to-date PCA response matrix. This work was supported in part by the Netherlands Organization for Scientific Research (NWO) under grant PGS 78-277 and by the Netherlands Foundation for Research in Astronomy (ASTRON). M. M. is a fellow of the Consejo Nacional de Investigaciones Científicas y Técnicas de la República Argentina. J. v. P. acknowledges support from the National Aeronautics and Space Administration through contract NAG 5-3269.
Barret, D., McClintock, J. E., \& Grindlay, J. E. 1996, ApJ, 473, 963

Belloni, T., Méndez, M., King, A. R., van der Klis, M., \& van Paradijs, J. 1997, ApJ, 479, L145

Bradt, H. V., Rothschild, R. E., \& Swank, J. H. 1993, A\&AS, 97, 355

Frank, J., King, A. R., \& Lasota, J.-P. 1987, A\&A, 178, 137

Greiner, J., Morgan, E. H., \& Remillard, R. A. 1996, ApJ, 473, 107

Hatchett, S., Buff, J., \& McCray, R. 1976, ApJ, 206, 847

Hjellming, R. M., \& Rupen, M. P. 1995, Nature, 375, 464

Kitamoto, S., Miyamoto, S., Tanaka, Y., Ohashi, T., Kondo, Y., Tawara, Y., \& Nakagawa, M. 1984, PASJ, 36, 731

Kitamoto, S., Miyamoto, \& Yamamoto, T. 1989, PASJ, 41, 81

Kuulkers, E., Belloni, T., Méndez, M., van der Klis, M., Wijnands, R., \& van Paradijs, J. 1997a, IAU Circ. 6637

Kuulkers, E., Parmar, A. N., Kitamoto, S., Cominsky, L. R., \& Sood, R. K. 1997b, MNRAS, 291, 81

Kuulkers, E., van der Klis, M., \& Parmar, A. N. 1997c, ApJ, 474, L47

Leahy, D. A. 1997, MNRAS, 287, 622

\section{REFERENCES}

Leahy, D. A., Yoshida, A., \& Matsuoka, M. 1994, ApJ, 434, 341

Levine, A. M., Bradt, H., Cui, W., Jernigan, J. G., Morgan, E. H., Remillard, R., Shirey, R. E., \& Smith, D. A. 1996, ApJ, 469, L33

Marshall, F. E., Mushotzky, R. F., Petre, R., \& Serlemitsos, P. J. 1993, ApJ, 419,301

Mitsuda, K., et al. 1984, PASJ, 36, 741

Orosz, J. J., \& Bailyn, C. D. 1997, ApJ, 477, 876; erratum, 482, 1086

Parmar, A. N., \& White, N. E. 1988, Mem. Soc. Astron. Italiana. 59, 147

Parmar, A. N., White, N. E., Giommi, P., \& Gottwald, M. 1986, ApJ, 308, 199

Remillard, R., \& Canizares, C. R. 1984, ApJ, 278, 761

Saraswat, P., et al. 1996, ApJ, 463, 726

Ueda, Y., Inoue, H., Tanaka, Y., Ebisawa, K., Nagase, F., Kotani, T., \& Gehrels, N. 1998, ApJ, 492, 782

van der Hooft, F., Heemskerk, M. H. M., Alberts, F., \& van Paradijs, J. 1998, A\&A, 329, 538

Zhang, S. N., et al. 1997, ApJ, 479, 381 